\title{
RTSM (Randomization and Trial Supply Management) for Early Phase Studies
}

\author{
Malcolm Morrissey ${ }^{*}$, Richard Roberts, Kurt Lumsden and Damian McEntegart
}

Perceptive Informatics, Meadow Grove, Nottingham, NG2 3HF, UK

\begin{abstract}
Phase I and early Phase II studies generally have the aim of establishing the safety, tolerability and pharmacokinetics of one or more doses and formulations. Traditionally many of these studies have tended to be conducted at a single site so that the randomization, dosing, dispensing, blood sampling procedures and progression between successive cohorts could be tightly managed.

Allowing competitive recruitment may be particularly important in patient studies in populations where it is difficult to recruit. RTSM (Randomization and Trial Supply Management) technology allows management of the recruitment to each cohort which would be difficult, if not impossible, to coordinate with many centers.

Examples are given in this letter to show how RTSM technology can be used to manage randomization, cohort progression and dosing in multicentre early phase trials.

Additionally, a sample survey of the incidence and types of early phase cohort studies performed by Perceptive Informatics was undertaken. The survey of our database showed that many early phase studies are conducted in multiple sites and countries with fairly low numbers of patients per site; this reflects the need for speed on the critical path for drug development.
\end{abstract}

Keywords: Cohort, RTSM (randomization and trial supply management), replacement randomization.

\section{INTRODUCTION}

Randomization and Trial Supply Management (RTSM) systems use telephone and Interactive Voice Response (IVR) technology to manage randomization and study medication dispensing and inventories in many clinical studies. They originated in the $1990 \mathrm{~s}$ and according to one regulatory source are used in the majority of multicentre trials conducted by the pharmaceutical industry [1]. Complimentary systems that allow dual telephone and web access are now commonplace. In addition to managing randomization and trial supplies, these systems are useful to monitor real time recruitment, manage emergency treatment code-breaking and perform calculations to ensure accuracy of dosing [2]. Other complex applications have been described [3]. But to date nothing has been written about their use in the management of early phase cohort studies and this is the subject of the paper.

Phase I and early Phase II studies generally have the aim of establishing the safety, tolerability and pharmacokinetics of one or more doses and formulations. More specifically the goals of a Phase I study are to determine the maximum tolerated dose (MTD) of a drug for a specified mode of administration and to characterise the most frequent and dose limiting toxicities. Designs used include the $3+3$ design, up and down designs and the continual reassessment method [4]. A characteristic of many such studies is that patients are entered in a staggered fashion to different cohorts where

*Address correspondence to this author at the Perceptive Informatics, Meadow Grove, Nottingham, NG2 3HF, UK; Tel: +44 (0)115 844 3909; Fax: +44 (0)115955 7555; E-mail: Malcolm.Morrissey@perceptive.com progression to subsequent cohorts is determined by the performance of the previous cohort. In some studies the dose progression is fixed in advance and the dose planned for each cohort is known. In other studies the dose step used for subsequent cohorts may be determined by the number of toxicities observed in the previous cohort and future doses are not known in advance. Often there is a pause between enrolments into successive cohorts as the performance of the dose used in the previous cohort is evaluated by a Data Monitoring Committee.

Traditionally many of these studies have tended to be conducted at a single site so that the randomization, dosing, dispensing, blood sampling procedures and progression between successive cohorts could be tightly managed. But given the need to accelerate drug development, there is a trend to sponsors using RTSM technology to enable these studies to be opened up to more centers; often these centers are located in different countries. Allowing competitive recruitment may be particularly important in patient studies in populations where it is difficult to recruit. RTSM technology allows management of the numbers recruited to each cohort which would be difficult, if not impossible, to coordinate with many centers. Similarly RTSM technology allows the management of progression between cohorts which reduces the administration and communication effort involved if sites were being managed manually via traditional methods such as fax and email.

This paper is split into three sections. We will detail some typical RTSM functionality that may be built as standard modules/functionality to aid the management of early phase cohort studies. We will then describe some 
advanced features that are used less regularly. Finally, a sample survey of the incidence and types of early phase cohort studies performed by Perceptive Informatics was undertaken. The results of this survey are described.

\section{TYPICAL RTSM (RANDOMIZATION AND TRIAL SUPPLY MANAGEMENT) FUNCTIONALITY}

When considering how to manage an early phase cohort study, it might be initially thought that the flexibility required is too complex to allow production of a generic prevalidated RTSM system. However, it is possible to produce validated configurable modules which allow an RTSM system to handle the most commonly requested cohort functionality. At Perceptive Informatics there are standard modules available for: opening and closing of cohorts, recruitment caps and replacement randomizations. Hence management of an early phase cohort study through RTSM can be set up in a timely manner by parameterizing a prevalidated system. During the recruiting phase of a clinical trial, movement between cohort randomization schedules and their associated medication schemes can be controlled through simple IVR/IWR transactions made inside the RTSM system.

Early phase studies may typically require recruitment of a small number of subjects across many sites. An RTSM system can behave as the 'gate keeper' in studies with 'stop start' recruitment. Recruitment into a fixed cohort of subjects can therefore be controlled centrally across multiple sites. Administrator IVR/Web transactions inside the RTSM system can be designed to open/close cohort recruitment, as well as set recruitment limits and define dose levels.

In a randomized cohort study, each cohort can be associated with a separate randomization schedule inside the RTSM system. Alternatively a single schedule stratified by cohort can be used with the treatments denoted by generic identifiers with no need to identify the dose e.g. "TEST" and "PLACEBO". IVR/Web transactions can be tailored to a particular protocol. For example, if a study has 3 potential cohorts, then a way of activating or prematurely deactivating a cohort may be required.

In Fig. (1) an RTSM call flow (transaction map) could allow the study wide opening or closing of a cohort, as well as setting the dose level for a cohort. This is an example of how a simple management call could be placed to manage study wide recruitment into a cohort study. The subsequent randomization transaction made inside the active cohort will use the appropriate dose level and recruitment limit information. Additional functionality may be required by a particular sponsor and transactions can include extra functionality. For example, a pre-specified recruitment target may not be possible during the design phase, and a recruitment target may need to be specified during the activation of a cohort.

Each cohort may have a fixed recruitment target and an RTSM system can enforce that limit. But if a hard cohort recruitment limit is employed, then withdrawals can affect any subsequent data analysis. If a patient withdraws before completing all treatment visits (or before completing a desired follow-up period), then replacement functionality may be required to achieve treatment assignment balance within a cohort. The most common requirement is that patients are replaced on a "like for like" basis i.e. the replacement patient receives the same treatment as the patient who has withdrawn.

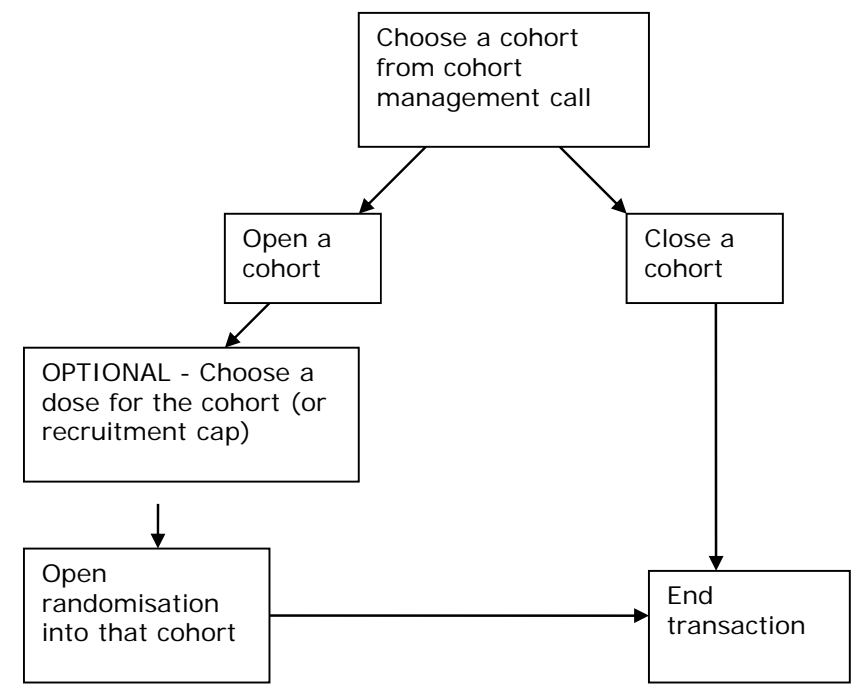

Fig. (1). Example of an IVR cohort activation call flow.

\section{SIMPLISTIC REPLACEMENT PROCEDURE IN TRADITIONAL STUDY}

In randomized double-blind studies managed without automated methods, medication supplies are provided in kits which contain the patient number on the kit label. Replacement can thus be effected by having a set of corresponding parallel reserve kits with the same numbering system e.g. same numbering system suffixed by $R$. The investigator will have been instructed that if a patient is to be replaced, then he/she should allocate the patient the corresponding R-numbered pack. Notice that this can only be done once, it is wasteful of supplies and may have unbinding implications.

Alternatively, the benefit of RTSM is that automated replacement can be achieved in a blinded manner.

\section{REPLACEMENT THROUGH AUTOMATED RANDOMIZATION SEQUENCE MANIPULATION}

To allow for replacement randomizations we need to consider the randomization list design. Inside the randomization schedule, it is possible to have the spare randomization code below the main cohort randomization records that are reserved for replacement subject randomizations only. These records could be unavailable in the RTSM system at the start of the study and a replacement randomization entry in the spare records could be freed up each time a withdrawal occurs (Fig. 2). If a matching treatment group entry is freed up, then we can guarantee a like for like treatment group replacement will take place when the freed up entry is used. The option here could be to close cohort recruitment only when we have used all available randomization records. This will include the replacement records freed up during the study which will be used last.

Within the RTSM databases there would be a full audit trail and traceability so that it is clear which patient being replaced is responsible for the 'Unavailable' entry being 
freed-up. Stratification can also be accounted for in the replacement randomization list. However, if a stratum specific replacement is required, then this may delay completion of a cohort as we wait for an appropriate subject to enter the study.

\begin{tabular}{|c|c|c|c|c|}
\hline & Numb & Trt & Status & Action \\
\hline \multirow{6}{*}{ 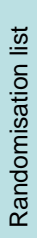 } & 0001 & A & Used & \\
\hline & 0002 & $\mathrm{C}$ & Used & \\
\hline & 0003 & $\mathrm{D}$ & Used & Withdrawn \\
\hline & 0004 & $E$ & Used & \\
\hline & 0005 & B & A & \\
\hline & \multicolumn{2}{|l|}{$\ldots$} & & \\
\hline \multirow{6}{*}{ 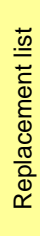 } & 1001 & $A$ & $\mathrm{R}$ & \\
\hline & 1002 & C & $\mathrm{R}$ & \\
\hline & 1003 & $\mathrm{D}$ & A & Freed up for replacement \\
\hline & 1004 & $E$ & $\mathrm{R}$ & \\
\hline & 1005 & B & $\mathrm{R}$ & \\
\hline & \multicolumn{2}{|l|}{$\ldots$} & & \\
\hline
\end{tabular}

Fig. (2). Example of a randomization list which can allow replacement.

Additionally, if it is expected that subjects may discontinue many times, a facility for multiple replacements can be considered. For example, two replacement lists could be employed to allow a randomization number to be replaced twice as a maximum (Table 1). In this case Record 1206 would be used to directly replace record 1106 if the subject receiving that entry withdraws. Likewise, Record 1106 would have been used as a Replacement for Record 1006.

Table 1. Example Randomization Number Format to Allow Replacement

\begin{tabular}{|c|c|c|}
\hline \multirow{2}{*}{ Cohort } & $\begin{array}{c}\text { Original or Replacement } \\
\text { Randomization List }\end{array}$ & $\begin{array}{c}\text { Randomization } \\
\text { Number Range }\end{array}$ \\
\hline \hline \multirow{3}{*}{1} & Original & $1001-1048$ \\
\cline { 2 - 3 } & Replacement list 1 & $1101-1148$ \\
\cline { 2 - 3 } & Replacement list 2 & $1201-1248$ \\
\hline \multirow{2}{*}{2} & Original & $2001-2048$ \\
\cline { 2 - 3 } & Replacement list 1 & $2101-2148$ \\
\cline { 2 - 3 } & Replacement list 2 & $2201-2248$ \\
\hline
\end{tabular}

The choice of whether to allow replacement can be left up to the study manager who simply uses the cohort management call to increase the recruitment target or it can be automated based on progress within the study e.g. an extra subject is needed if the investigator makes a withdrawal call.

\section{OTHER USEFUL TECHNIQUES CONTROLLABLE THROUGH RTSM FUNCTIONALITY}

In addition to the standard functionality previously discussed, it is always possible to incorporate other functionality on a bespoke programming basis. Advanced or less common functionality that Perceptive Informatics has experience with includes the following aspects of early phase studies:

\section{Time Interval Constraints}

Staggered dosing within a cohort e.g. 48 hours between successive subjects could be a requirement of a study design. This may be due to a safety concern of the drug being investigated. An observation period may be required in between randomizations to a particular active dose/treatment arm. As part of the time interval constraint, it may be that all recruitment stops study wide for the designated period, or that only the particular treatment arm closes for the desired time period. If the latter option is required, then 'Forcing' can be utilized to 'skip' randomization entries of the temporarily closed treatment arm. This would not be forcing of the randomization due to lack of medication, but forcing to change the randomization design. The option would be available to allow back filling once the 'observation arm' is taken off hold.

\section{Flexible Access to Any Cohort at a Study Level}

Flexible opening and closing cohort calls can aid study management. Where required, we have implemented the functionality to re-open previously closed cohorts. This may be required to allow replacement of a subject in a previously closed cohort. Or to allow a premature transition to the next cohort, that may be due to positive results of an interim analysis for which a cohort of a higher dose is now required. Another reason could be a safety concern, for which a cohort of a lower dose is now required.

\section{Over-Recruitment Avoidance}

Ability to have automatic temporary suspension of screening when the total of those currently in screening and randomized/enrolled is equal to the randomization/enrolment target; screening automatically re-opens with appropriate site notification if one or more of subjects currently in screening drops out prior to randomization meaning that the target has not been reached.

\section{Adaptive Dosing}

Adaptive dosing where the dose for successive cohorts is determined by the analysis of previous cohorts. The dosing for the next cohort can be implemented as part of the open cohort call. Altered dosing frequency can be accommodated within the call if the possibilities can be defined up-front. Medication release can be addressed as part of the same call or as an earlier call.

\section{Establishing Minimum Effective Dose}

In certain indications sequential cohort studies can be used to establish the minimum effective dose. A specific example is the up and down design where the dosing to be used in successive cohorts is determined by the collection of the subject responses via the RTSM system. This type of design is suited to single dosing studies where the subject response is rapidly observed and readily communicated to the RTSM system via the patient or investigator e.g. headache or migraine. For further details of these designs are given by Hall, Meier and Diener [5]. Patients are recruited in successive cohorts with the dose of the next cohort determined according to a simple rule. In a trial by Oleson [6], groups of 6 patients presenting with moderate to severe migraine were treated with 4 patients randomized to a dose of the experimental treatment and 2 to receive placebo. The 
dose in the next group was decreased if at least 3 of the 4 patients had a response; otherwise the dose was increased. At the highest or lowest dose, the rule was modified to avoid treatment outside the target range. The up and down process was to be terminated when one of the doses satisfied prespecified selection criteria. Note the Olesen trial was not managed by RTSM but we have managed similar trials using the RTSM system. The Olesen trial [6] is discussed as it is published material.

\section{RESULTS OF SURVEY}

\section{Survey Details}

We examined our database to determine the incidence, types of studies being conducted and functionality employed using Perceptive Informatics. We determined the total number of studies by an automated scan for the phrase 'Cohort' in our database which at the time of the scan contained approximately 2800 studies that had been put live. This gave us a total of 258 cohort studies. We randomly sampled 50 of these and classified them according to type and functionality.

Some studies had two distinct stages, an earlier dose finding cohort study to determine the dose to be studied in a subsequent phase with a larger number of patients. We term this a seamless Phase I-II study. To avoid distortion of the patient and site numbers we have only considered the dose finding phase in our calculations.

Perhaps as expected we did not find any cohort studies that enrolled healthy volunteers. Such studies are handled in dedicated units without the need for automated management. Instead all the studies involved patients with a defined disease. The diseases involved are tabulated in Table 2. Of note is the large proportion of diseases that might be classed as serious according to the Food and Drug Administration (FDA) guide for consumers [7] to participating in clinical trials ( 46 out of 50 studies sampled).

The number of cohorts, sites and countries involved in the studies are given in Figs. (3-7). Fig. (3) shows that 86\% of sampled studies had more than 2 cohorts. It was observed that $58 \%$ of studies in the sample included more than 10 sites in Fig. (4). Moreover 68\% of studies included sites from more than 1 country in Fig. (5). This might not be indicative of all cohort studies in the industry, but of the type of study run with RTSM systems, where central management of many sites is required to meet recruitment targets in patient populations where recruitment is challenging.

We calculated the median value of the numbers of patients per site at the trial planning stage as 4.80 (range 0.64 to 105.00). We further divided this figure by the number of countries as this gives an indication of the logistical effort involved by the sponsor; the median value was 3.34 (range $=0.11$ to 105.00 ). Histograms of the distributions are given in Figs. $(6,7)$ respectively. Six studies had an average recruitment of less than a single patient per site per country which represents a substantial effort per patient when one considers the logistical considerations including medication distribution and translations. The low numbers involved per site and country are less than we see generally indicating that these are difficult to recruit populations and that there must be a need for speed to get these studies completed; presumably they are on the critical path and a rate limiting factor before more extensive Phase II trials can commence.

Table 2. Distribution of Indications Observed in Sample Survey

\begin{tabular}{|c|c|c|}
\hline Serious & Indication Abbreviation & Total \\
\hline \multirow{4}{*}{ No } & Atopic Dermatitis & 1 \\
\hline & Dengue fever & 1 \\
\hline & Dry eye disease & 1 \\
\hline & Genital Warts & 1 \\
\hline \multirow{23}{*}{ Yes } & Alzheimer's & 5 \\
\hline & Atrial Fibrillation & 1 \\
\hline & Bleeding & 1 \\
\hline & Brain Injury & 1 \\
\hline & Cancer & 10 \\
\hline & Chronic Hepatitis C & 2 \\
\hline & Coronary Artery Bypass Surgery & 1 \\
\hline & Cystic fibrosis & 1 \\
\hline & Diabetes & 2 \\
\hline & Fungal infections (systemic) & 1 \\
\hline & Glioblastoma & 1 \\
\hline & Heart Failure & 1 \\
\hline & Hematopoietic stem cell transplant. & 1 \\
\hline & Hyperlipidemia & 1 \\
\hline & Lupus & 1 \\
\hline & Malaria & 1 \\
\hline & MI & 1 \\
\hline & Multiple sclerosis & 2 \\
\hline & Rheumatoid arthritis & 6 \\
\hline & Sepsis & 1 \\
\hline & Stroke & 3 \\
\hline & Ulcerative Colitis & 1 \\
\hline & Venous thromboembolism & 1 \\
\hline Serious Total & & 46 \\
\hline Grand Total & & 50 \\
\hline
\end{tabular}

Code break calls can be programmed within the RTSM system to be made by one or more of the investigator or study leader or global drug safety department level. Automated code break calls provide greater security and a superior audit trail as compared to other methods (code breaker envelopes, 24 hour manned telephone line etc.). Preservation of the blind is particularly important in cohort studies with small numbers of patient per cohort and any impact of selection bias or assessment bias caused by knowledge of individual patient's treatment would be magnified given the small numbers in the study. $84.0 \%$ of the studies were randomized, double blind studies and of 
these $76.2 \%$ used RTSM code break facility. In those randomized studies where a code break facility was not employed, emergency code breaking was either handled by the sponsor or by code breaker envelopes. The latter is not always a satisfactory solution - for instance Schulz and Grimes [8] detail the lengths that some investigators will go to illicitly break the blind.

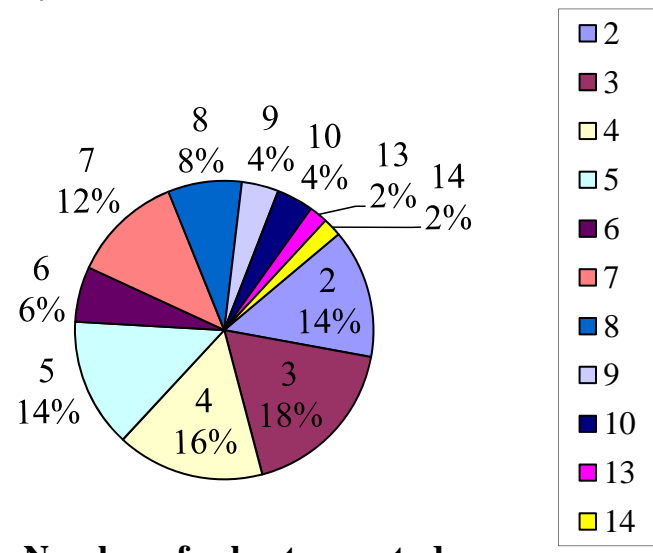

Number of cohorts per study

Fig. (3). Pie chart showing the number of cohorts per study surveyed.

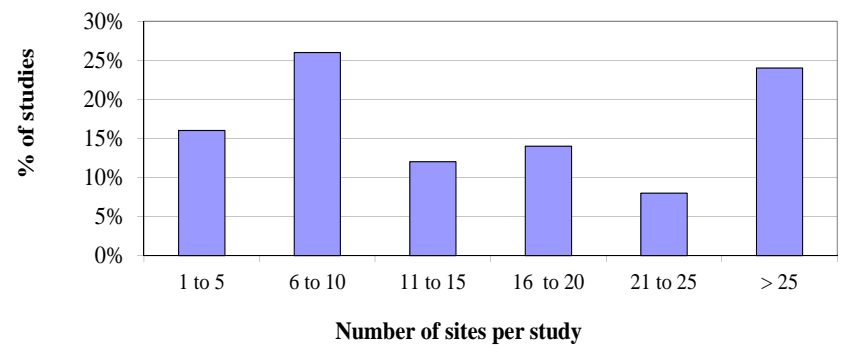

Fig. (4). Distribution of the number of sites per study surveyed.

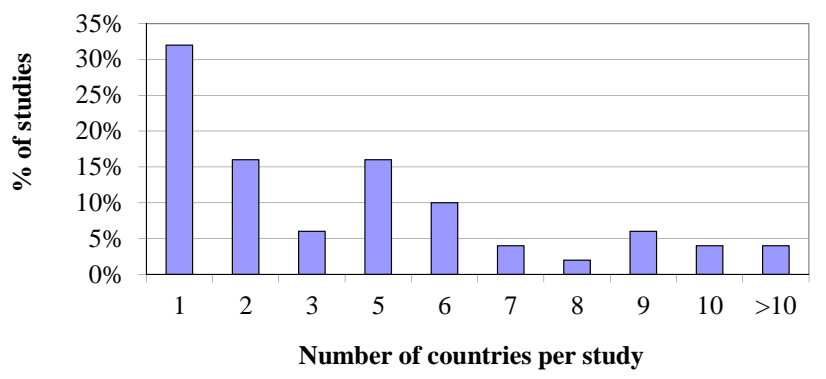

Fig. (5). Distribution of the number of countries per study surveyed.

Interactive web response (IWR) functionality is proving very popular in studies managed by electronic systems. It allows the users to see all the administrative options on screen and this simplifies the interface in many cases. Selections can be made from drop down lists if desired and all IVR functionality can be mirrored in IWR systems. 53\% of the sample studies were built with IWR as well as IVR.

A related type of study to Phase I/IIa dose finding designs are those where successive cohorts are defined by age, weight or disease severity. Here the sequential cohorts are typically started in older, heavier or less ill patients and then if tolerability is satisfactory, successive cohorts for younger, lighter or more severely ill patients are opened. A small number of studies made use of specialized features of the RTSM system. Two studies used minimization with a random element to perform the randomization within each cohort [9]. The use of this technique has become somewhat controversial in later Phase studies [10], but it is a useful technique to achieve balance on important prognostic factors in studies with small numbers of patients. It can be used to achieve balance between a specific dose and placebo within the cohort and simultaneously between all doses of the experimental treatment and placebo across the cohort.

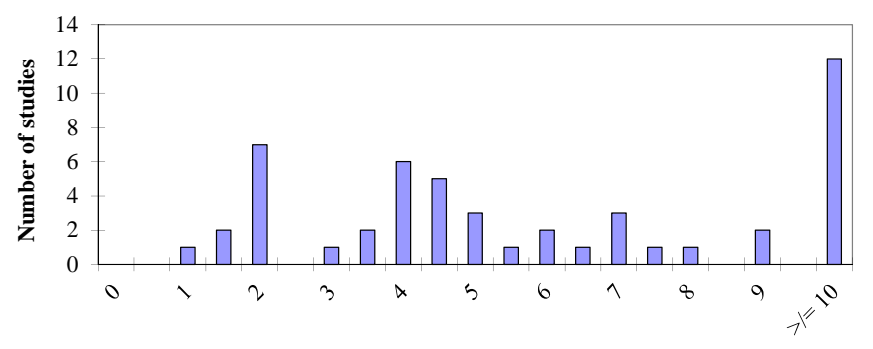

Subjects per site

Fig. (6). Distribution of the number of subjects per site inside each study surveyed.

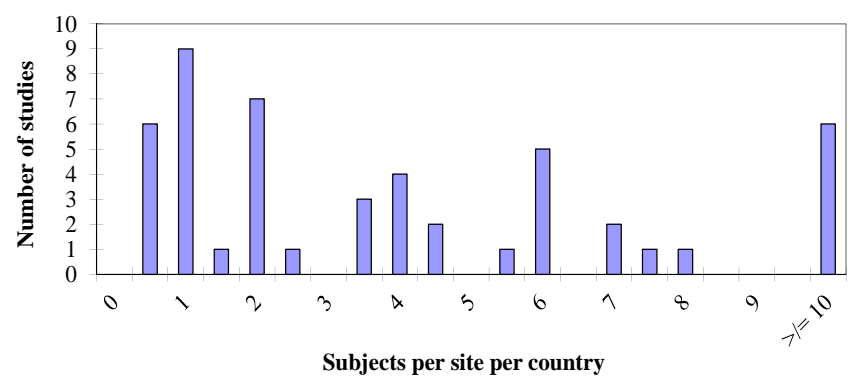

Fig. (7). Distribution of the number of subjects per site per country inside each study surveyed.

Two studies used the RTSM system to capture patient responses. The use of electronic Patient Reported Outcome methods has to date been fairly limited in early phase studies but we can expect their use to increase as FDA focus on data quality and avoidance of missing data as outlined in their recent guidance [11].

\section{CONCLUSIONS}

RTSM functionality is increasingly used to manage studies which require control of entry into sequential cohorts. The electronic systems allow superior functionality to traditional non-automated methods which are restrictive and in particular limit the number of participating sites. Our survey of our database has shown that many cohort studies are conducted in multiple sites and countries with fairly low numbers of patients per site; this reflects the need for speed given that these studies are often on the critical path for drug development.

\section{CONFLICT OF INTEREST}

The authors are all employed by Perceptive Informatics, a provider of RTSM and clinical systems. 


\section{REFERENCES}

[1] Smith F. A regulatory perspective of the statistician's role in the data verification and inspection process. Biopharm Rep 2009; 16(1): 2-8.

[2] Byrom B. Using IVRS in clinical trial management. Appl Clin Trials 2002; 11(10): 36-42.

[3] Byrom B, McEntegart D, Morrissey M. Complex problems, simple solutions. GCPj 2008; 15(7): 15-8.

[4] Chevret S, Ed. Statistical methods for dose-finding experiments. New York: Wiley 2006.

[5] Hall DB, Meier U, Diener H-C. A group sequential adaptive treatment assignment design for proof of concept and dose selection in headache trials. Contemp Clin Trials 2005; 26: 349-64.

[6] Olesen J, Diener H-C, Husstedt IW, et al. Calcitonin gene-related peptide receptor antagonist BIBN $4096 \mathrm{BS}$ for the acute treatment of migraine. N Engl J Med 2004; 350: 1104-10.

[7] Food and Drug Administration. FDAMA section 113: status report on implementation, Appendix H: List of serious and non-serious diseases and conditions [guide on the internet]. [Accessed 2011 October 12]. Available from: http://www.fda.gov/forconsu mers/byaudience/forpatientadvocates/participatinginclinicaltrials/uc m148651.htm

[8] Schulz KF, Grimes DA. Allocation concealment in randomized trials: defending against deciphering. Lancet 2002; 359: 614-8.

[9] Scott NW, McPherson GC, Ramsay CR, Campbell MK. The method of minimization for allocation to clinical trials: a review. Control Clin Trials 2002; 23: 662-74.

[10] Buyse M, McEntegart D. Achieving balance in clinical trials, an unbalanced view from EU regulators. App Clin Trials 2004; 13(5): 36-40.

[11] Food and Drug Administration. Guidance for industry: patientreported outcome measures: use in medical product development to support labeling claims (final), December 2009. [guide on the internet]. [Accessed 2011 October 12]. Available from: http://ww w.fda.gov/downloads/Drugs/GuidanceComplianceRegulatoryInfor mation/Guidances/UCM193282.pdf

(C) Morrissey et al.; Licensee Bentham Open.

This is an open access article licensed under the terms of the Creative Commons Attribution Non-Commercial License (http://creativecommons.org/licenses/by$\mathrm{nc} / 3.0 /$ ) which permits unrestricted, non-commercial use, distribution and reproduction in any medium, provided the work is properly cited. 